\title{
PERFORMANCE ESTIMATION OF ADHOC ROUTING PROTOCOLS WITH NS2 SIMULATOR
}

\author{
Syeda Kausar Fatima, Dr. Syeda Gauhar Fatima, Dr.Syed Abdul Sattar and Syed Mohd \\ Ali \\ Research Scholar JNTUH, Hyderabad, India
}

\begin{abstract}
ABTRACT
A mobile adhoc network is a collection of wireless mobile nodes dynamically creating a temporary network without usage of any prevailing network infrastructure or consolidated management. Several routing protocols like Dynamic Source Routing (DSR), Adhoc On-Demand Distance Vector Routing (AODV) and Destination Sequenced Distance-Vector (DSDV) have been implemented. In this paper, an attempt has been made to compare the performance of two prominent on-demand reactive routing protocols for mobile ad hoc networks: DSR and AODV, along with the traditional proactive DSDV protocol. A simulation model with MAC and physical layer models is used to study interlayer interactions and their performance inferences. The On-demand protocols, AODV and DSR perform better than the table-driven DSDV protocol. Although DSR and AODV share similar on-demand performance, the differences in the protocol mechanics can lead to significant performance differentials. A variation of workload and scenarios, as characterized by mobility, load and size of the ad hoc network were simulated. The performance analysis is evaluated by means of varying network load, mobility, and network size. These simulations are conceded out based on the Rice Monarch Project that has made considerable additions to the NS-2 network simulator to track adhoc simulations.
\end{abstract}

Keywords: Performance, Analysis, Ad Hoc Network, AODV, DSR, DSDV, NS2.

Cite this Article: Syeda Kausar Fatima, Dr. Syeda Gauhar Fatima, Dr.Syed Abdul Sattar and Syed Mohd Ali, Performance Estimation of Adhoc Routing Protocols with Ns2 Simulator, International Journal of Advanced Research in Engineering and Technology, 10(2), 2019, pp. 215-223.

http://iaeme.com/Home/issue/IJARET?Volume=10\&Issue $=2$

\section{INTRODUCTION}

Wireless networking is a developing technology that permits users to access information and services electronically, regardless of their geographic location. Wireless networks can be classified in two types. 


\subsection{Infrastructure Networks}

Infrastructure network comprises of a network with fixed and wired gateways. A mobile host communicates with a bridge in the network (called base station) within its communication range. The mobile unit can travel geographically while it is communicating. When it goes out of range of one base station, it associates with new base station and starts communicating through it. This is called handoff. In this method the base stations are stationary.

\subsection{Infrastructure Less (Adhoc) Networks}

In adhoc networks [5] all nodes are mobile and can be linked dynamically in a random manner. As the range of each host's wireless transmission is restricted, so to communicate with hosts outside its transmission range, a host needs to enlist the aid of its nearby hosts in forwarding packets to the destination. So all nodes of these networks behave as routers and take part in discovery and maintenance of routes to other nodes in the network. Adhoc networks are suitable in emergency search and rescue operations, meetings or conventions in which persons wish to rapidly share information, and data acquirement operations in unfriendly topography. This adhoc routing protocols can be divided into two classifications:

Table Driven Routing Protocols: In table driven routing protocols, reliable and up-to-date routing information to all nodes is preserved at each node.

On-Demand Routing Protocols: In On-Demand routing protocols, the paths are formed as and when essential. When a source needs to send to a destination, it raises the route discovery mechanisms to discover the path to the destination.

\section{AD-HOC ROUTING PROTOCOLS DESCRIPTION DESTINATION- SEQUENCED DISTANCE-VECTOR}

The Destination-Sequenced Distance-Vector (DSDV) [3] Routing Algorithm is established on the idea of the traditional Bellman-Ford Routing Algorithm with certain improvements. Every mobile node maintains a routing table that lists all available destinations, the number of hops to reach the destination and the sequence number allocated by the destination node. The sequence number is used to distinguish stale routes from new ones and therefore avoid the creation of loops. The nodes occasionally transmit their routing tables to their immediate neighbors. A station also transmits its routing table if a significant change has occurred in its table from the last update sent. So, the update is both time-driven and event-driven. The routing table updates can be sent in two ways: a "full dump" or an incremental update. A full dump sends the full routing table to the neighbors and could span many packets whereas in an incremental update only those records from the routing table are sent that has a metric variation since the last update and it must fit in a packet. If there is space in the incremental update packet then those records may be incorporated whose sequence number has altered. When the network is pretty stable, incremental updates are sent to shun extra traffic and full dump are relatively infrequent. In a fast-changing network, incremental packets can grow big so full dumps will be more common.

\section{AD HOC ON-DEMAND DISTANCE VECTOR ROUTING (AODV)}

AODV [2] discovers routes on an as required basis via a similar route discovery process. However, AODV implements a very different mechanism to maintain routing information.

It uses traditional routing tables, one entry per destination. This is in contrast to DSR, which can maintain multiple route cache entries for each destination. Without source routing, AODV relies on routing table entries to broadcast an RREP back to the source and, subsequently, to route data packets to the destination. AODV uses sequence numbers maintained at each 
destination to determine freshness of routing information and to prevent routing loops. All routing packets carry these sequence numbers.

An important feature of AODV is the maintenance of timer-based states in each node, regarding utilization of individual routing table entries. A routing table record is expired if not used recently. A set of predecessor nodes is preserved for each routing table entry, representing the set of neighboring nodes which use that entry to route data packets. These nodes are notified with RERR packets when the next-hop link breaks. Each predecessor node, in turn, forwards the RERR to its own set of predecessors, thus effectively erasing all routes using the broken link. In contrast to DSR, RERR packets in AODV are intended to inform all sources using a link when a failure occurs. Route error propagation in AODV can be visualized conceptually as a tree whose root is the node at the point of failure and all sources using the failed link as the leaves.

\section{DYNAMIC SOURCE ROUTING (DSR)}

The main distinctive feature of DSR [4] is the use of source routing. That is, the sender knows the complete hop byhop route to the destination. These routes are stored in a route cache. The data packets carry the source route in the packet header. When a node in the adhoc network tries to send a data packet to a destination for which it does not already know the route, it uses a route discovery process to dynamically determine such a route. Route discovery works by flooding the network with route request (RREQ) packets. Each node receiving an RREQ rebroadcasts it, unless it is the destination or it has a route to the destination in its route cache. Such a node replies to the RREQ with a route reply (RREP) packet that is routed back to the original source. RREQ and RREP packets are also source routed. The RREQ builds up the path traversed across the network. The RREP routes itself back to the source by traversing this path backward. The route carried back by the RREP packet is cached at the source for future use. If any link on a source route is broken, the source node is notified using a route error (RERR) packet. The source removes any route using this link from its cache. A new route discovery process must be initiated by the source if this route is still needed. DSR makes very aggressive use of source routing and route caching.

\section{PERFORMANCE ANALYSIS SIMULATION ENVIRONMENT}

The simulation experiment is carried out in LINUX (FEDORA 6). The detailed simulation model is based on network simulator-2 (ver-2.31) [1], is used in the evaluation. The NS instructions can be used to define the topology structure of the network and the motion mode of the nodes, to configure the service source and the receiver, to create the statistical data track file and so on.

\section{TRAFFIC MODEL}

Continuous bit rate (CBR) traffic sources are used. The source-destination pairs are spread arbitrarily above the network. Only 512-byte data packets are used. The number of sourcedestination pairs and the packet sending rate in each pair is varied to change the obtainable load in the network.

\section{MOBILITY MODEL}

The mobility model uses the random waypoint model in a rectangular field. The field configurations used is: $500 \mathrm{~m} \times 500 \mathrm{~m}$ field with 50 nodes. Here, each packet starts its journey from a random location to a random destination with a randomly chosen speed (uniformly distributed between $0-20 \mathrm{~m} / \mathrm{s}$ ). Once the destination is reached, another random destination is targeted after a pause. The pause time, which affects the relative speeds of the mobiles, is varied. Simulations are run for 100 simulated seconds. Identical mobility and traffic scenarios are used 
across protocols to gather fair results. Mobility models were created for the simulations using 50 nodes, with pause times of $0,10,20,40,100$ seconds, maximum speed of $20 \mathrm{~m} / \mathrm{s}$, topology boundary of $500 \times 500$ and simulation time of 100 secs.

\section{PERFORMANCE METRICS [7] PACKET DELIVERY FRACTION}

The ratio of the data packets delivered to the destinations to those generated by the CBR sources is known as packet delivery fraction.

\section{AVERAGE END-TO-END DELAY}

Average end to end delay includes all possible delays caused by buffering during route discovery latency, queuing at the interface queue, retransmission delays at the MAC, and propagation and transfer times of data packets.

\section{NORMALIZED ROUTING LOAD}

The number of routing packets transmitted per data packet delivered at the destination. Each hop-wise transmission of a routing packet is counted as one transmission. The first two metrics are the most significant for best effort traffic. The routing load metric evaluates the efficiency of the routing protocol. Note, however, that these metrics are not completely independent. For example, lower packet delivery ratio means that the delay metric is evaluated with fewer samples. In the conventional perception, the longer the path lengths, the higher the probability of a packet drops. Thus, with a lower delivery ratio, samples are generally inclined in favor of smaller path lengths and therefore have less end to end delay.

\section{PERFORMANCE RESULTS}

For all the simulations, the identical movement models were used, the number of traffic sources was fixed at 20 , the maximum speed of the nodes is set to $20 \mathrm{~m} / \mathrm{s}$ and the pause time varied as $0 \mathrm{~s}, 10 \mathrm{~s}, 20 \mathrm{~s}, 40 \mathrm{~s}$ and $100 \mathrm{~s}$.

\section{PACKET DELIVERY FRACTION}

The On-demand protocols, DSR and AODV performed predominantly well, transporting over $85 \%$ of the data packets irrespective of mobility ratio.

\section{AVERAGE END-END PACKET DELAY}

The average end-to-end delay of packet delivery was higher in DSDV as compared to both DSR and AODV. In summary, both the On-demand routing protocols, AODV and DSR outperformed the Table-driven routing protocol; DSDV and the reasons are discussed later. Figures 1 and 2 highlight the relative performance of the three routing protocols. All of the protocols provide a greater proportion of the initiated data packets when there is little node mobility (i.e., at large pause time), converging to $100 \%$ delivery when there is no node mobility. Following, since both AODV and DSR did better, an effort was made to evaluate the performance difference among the two by changing the mobility arrangement and number of traffic sources. 


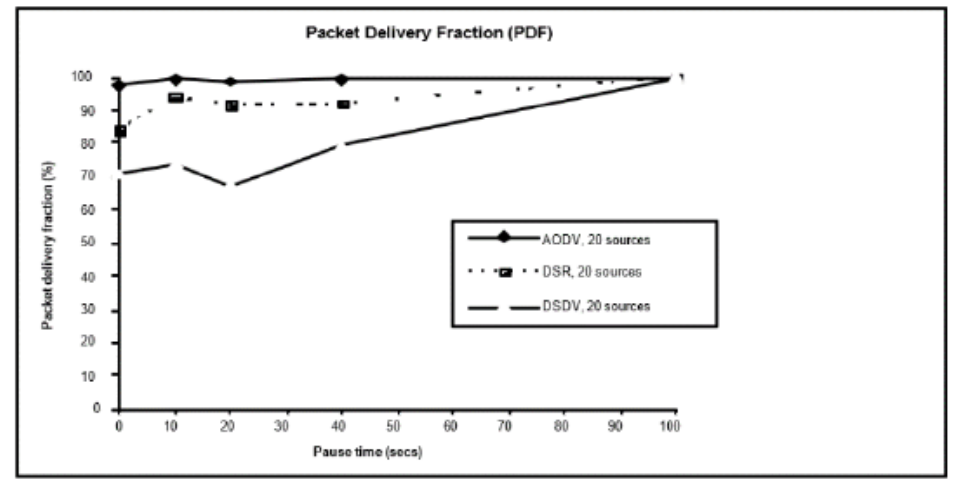

Figure 1 Packet Delivery Fraction (PDF) Vs Pause time

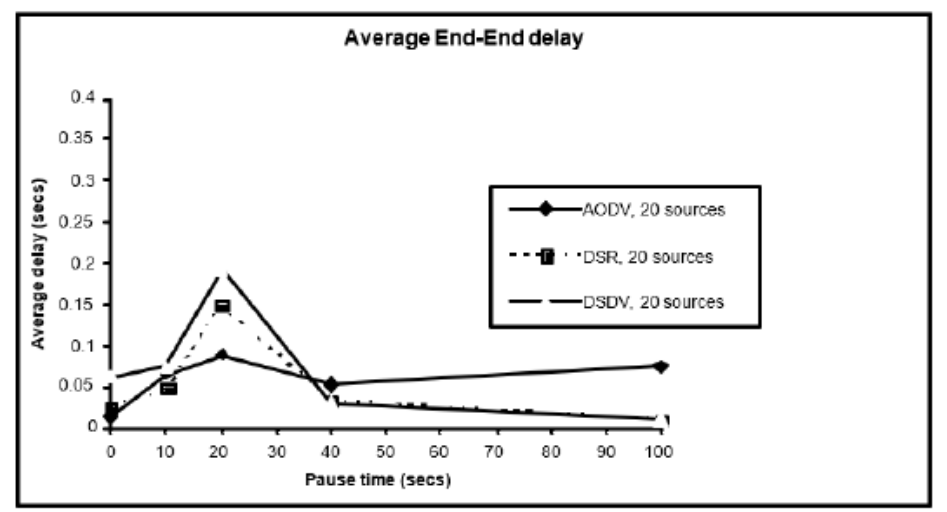

Figure 2 Average Delay Vs Pause Time

\section{VARYING MOBILITY AND NUMBER OF SOURCES TO ANALYZE THE PERFORMANCE MODIFICATION}

Now, again simulations were carried out with the number of traffic sources as 10, 20, 30 and 40.The pause time was varied as 0 (high mobility), 10, 20, 40, 100 (no mobility) and the packets were directed at a ratio of 4 packets/sec.

\subsection{Packet Delivery Fraction Comparison}

The packet delivery fractions [7] for DSR and AODV are alike with 10 sources (Fig. 3). However, with 20, 30 and

40 sources, AODV outperforms DSR by about 15 percent (Fig. 5 and 6) at lower pause times (higher mobility).

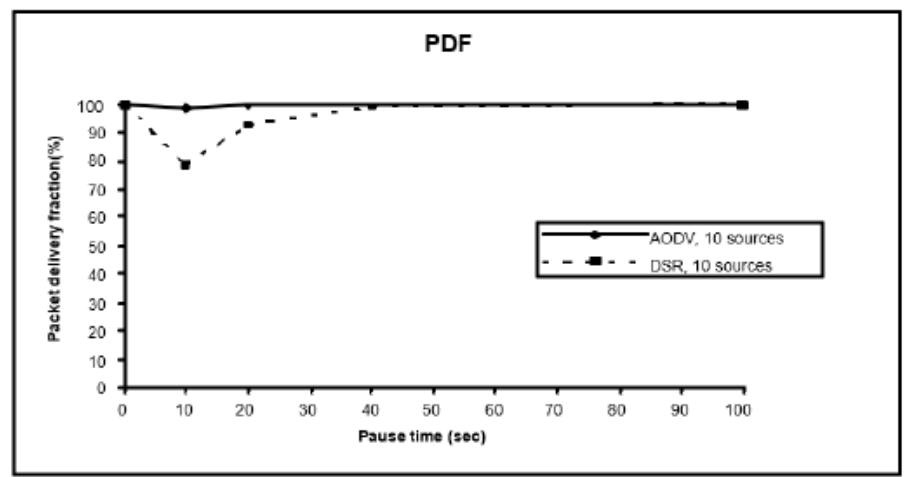

Figure 3 PDF for 10 Sources 

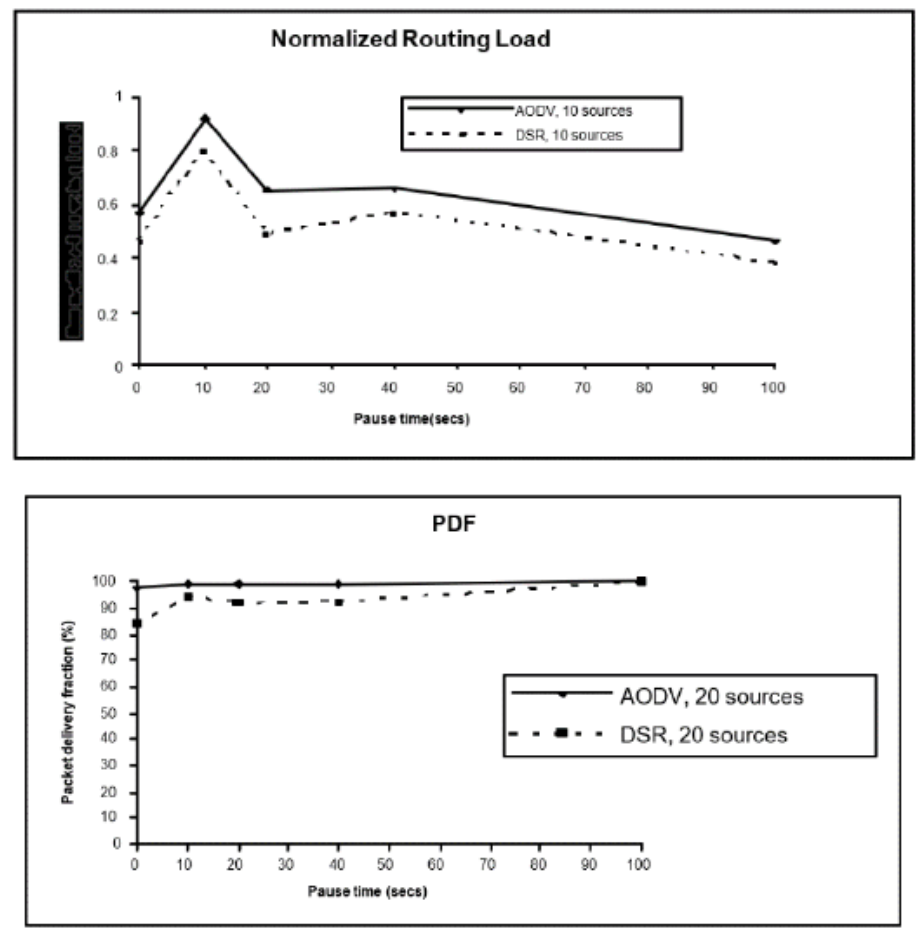

Figure 4 PDF for 20 Sources

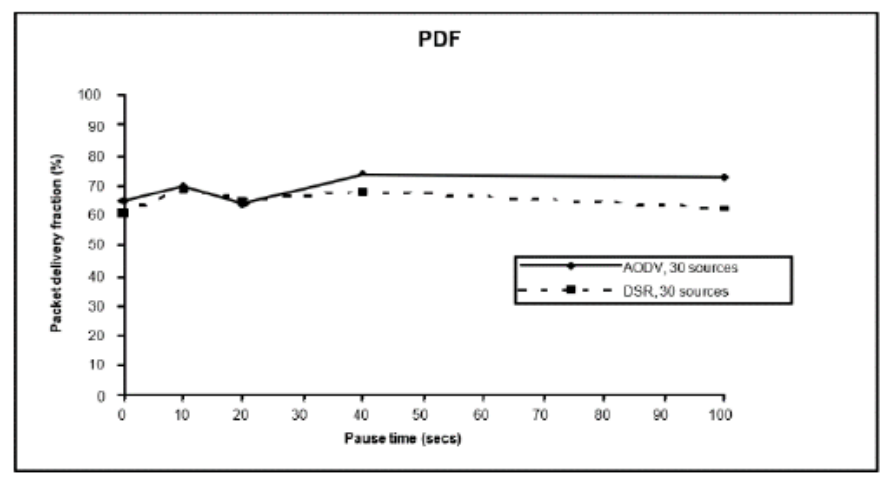

Figure 5 PDF for 30 Sources

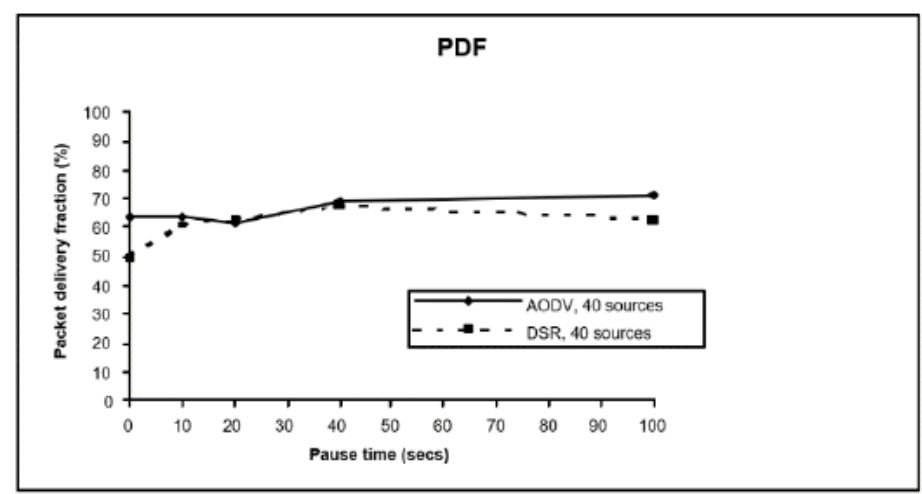

Figure 6 PDF for 40 Sources

\subsection{Normalized Routing Load Comparison}

In all cases, DSR demonstrates significantly lower routing load than AODV, with the factor increasing with a growing number of sources. In summary, when the number of sources is low, 
the performance of DSR and AODV is similar regardless of mobility. With large numbers of sources, AODV starts outperforming DSR for highmobility scenarios. As the data from the varying sources demonstrate, AODV starts outperforming DSR at a lower load with a larger number of nodes. DSR always demonstrates a lower routing load than AODV. The major contribution to AODV's routing over-head is from route requests, while route replies constitute a large fraction of DSR's routing overhead. Furthermore, AODV has more route requests than DSR, and the converse is true for route replies.

Figure 7 Normalized Routing Load for 10 Sources

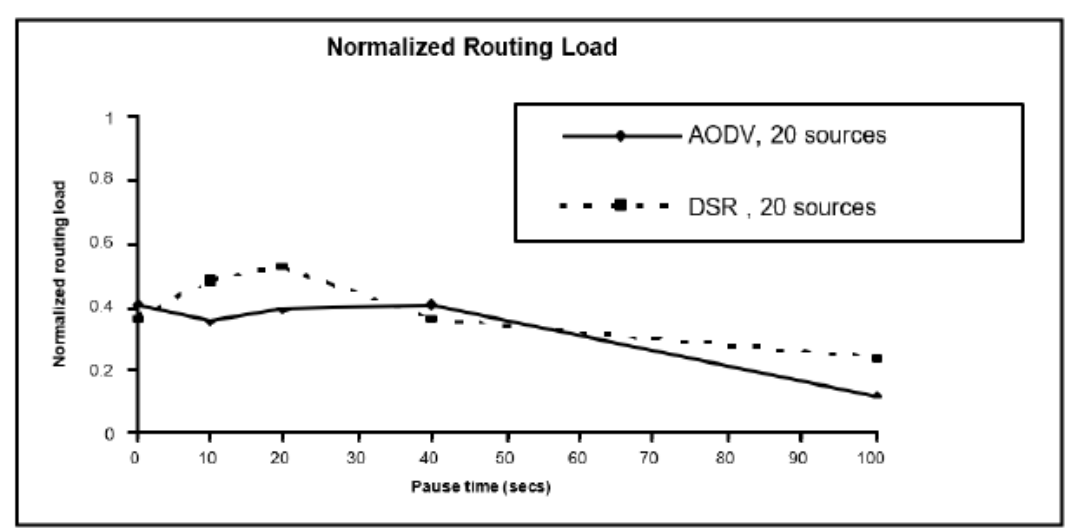

Figure 8 Normalized Routing Load for 20 Sources

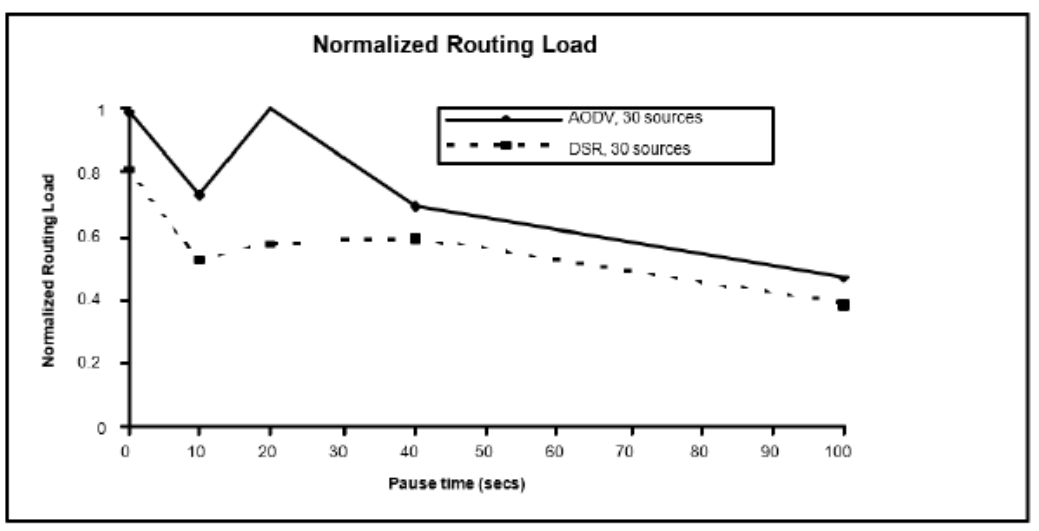

Figure 9 Normalized Routing Load for 30 Sources

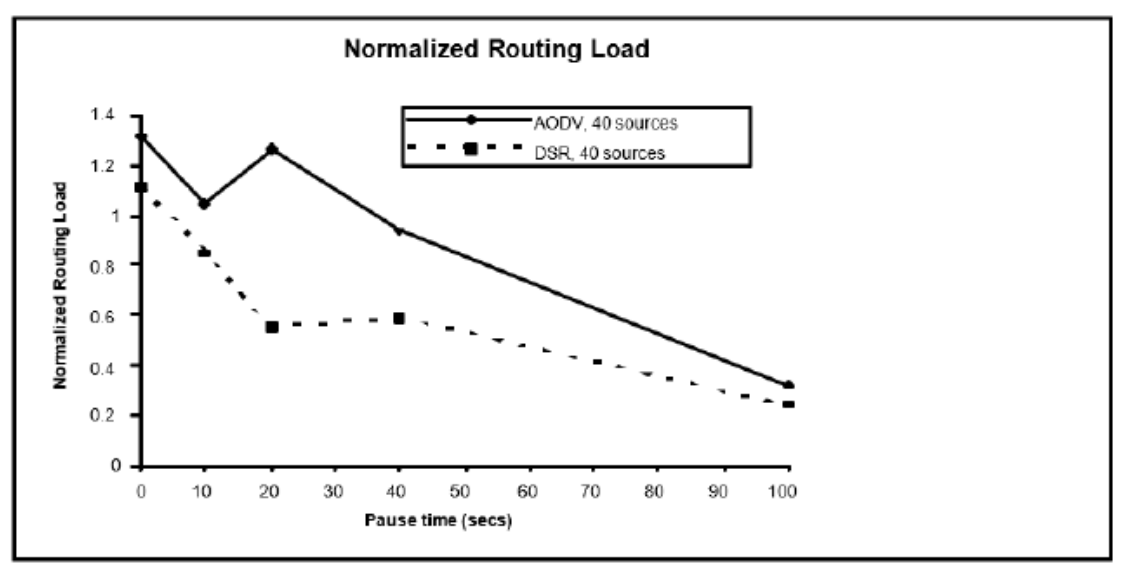

Figure 10 Normalized Routing Load for 40 Sources 


\section{PERFORMANCE ANALYSIS}

The simulation results bring out some important characteristic differences between the routing protocols. The presence of high mobility implies frequent link failures and each routing protocol reacts differently during link failures. The different basic working mechanism of these protocols leads to the differences in the performance. DSDV fails to converge below lower pause times. At higher rates of mobility (lower pause times), DSDV does poorly, dropping to a $70 \%$ packet delivery ratio. Nearly all of the dropped packets are lost because a stale routing table entry directed them to be forwarded over a broken link. As described in the earlier section, DSDV maintains only one route per destination and consequently, each packet that the MAC layer is unable to deliver is dropped since there are no alternate routes. For DSR and AODV, packet delivery ratio is independent of offered traffic load, with both protocols delivering between $85 \%$ and $100 \%$ of the packets in all cases. Since DSDV uses the table-driven approach of maintaining routing information, it is not as adaptive to the route changes that occur during high mobility. In contrast, the lazy approach used by the on-demand protocols, AODV and DSR to build the routing information as and when they are created make them more adaptive and result in better performance (high packet delivery fraction and lower average end-to-end packet delays). Next the simulation results of Normalized Routing Load graph with 30 and 40 sources that compare the performances of AODV and DSR lead us to the following conclusions.

\section{MOBILITY EFFECT}

In the presence of high mobility, link failures occur very frequently. Link failures trigger new route discoveries in AODV since it has at most one route per destination in its routing table. Thus, the frequency of route discoveries in AODV is directly proportional to the number of route breaks. The reaction of DSR to link failures in comparison is mild and causes route discovery less often. The reason is the abundance of cached routes at each node. Thus, the route discovery is delayed in DSR until all cached routes fail. But with high mobility, the chance of the caches being stale is quite high in DSR. Finally when a route discovery is originated, the large number of replies received in response is associated with high MAC overhead and cause improved interfering to data traffic. Hence, the cache staleness and high MAC overhead together result in significant degradation in performance for DSR in high mobility scenarios. In lower mobility scenarios, DSR regularly performs better than AODV, because the chances of find the route in one of the caches is much higher. However, due to the constrained simulation environment (lesser simulation time and lesser mobility models), the better performance of DSR over AODV couldn't be observed.

\section{ROUTING LOAD RESULT}

DSR almost always has a lower routing load than AODV.

This can be attributed to the caching strategy used by DSR. By virtue of aggressive caching, DSR is more likely to find a route in the cache, and hence resorts to route discovery less frequently than AODV.

\section{CONCLUSIONS}

This project compared the performance of DSDV, AODV and DSR routing protocols for adhoc networks using ns-2 simulator. DSDV uses the proactive table-driven routing strategy while both AODV and DSR use the reactive On demand routing strategy. Both AODV and DSR perform better under high mobility simulations than DSDV. High mobility outcomes in frequent link failures and the overhead involved in updating all the nodes with the new routing information as in DSDV is much more than that involved AODV and DSR, where the routes are created as and when required. DSR and AODV both use on-demand route discovery, but 
with different routing mechanics. In particular, DSR uses source routing and route caches, and does not depend on any periodic or timer-based activities. DSR exploits caching aggressively and maintains multiple routes per destination. AODV, on the other hand, uses routing tables, one route per destination, and destination sequence numbers, a mechanism to prevent loops and to determine freshness of routes. The general observation from the simulation is that for application-oriented metrics such as packet delivery fraction and delay AODV, outperforms DSR in more "stressful" situations (i.e., smaller number of nodes and lower load and/or mobility), with widening performance gaps with increasing stress (e.g., more load, higher mobility). DSR, however, consistently generates less routing load than AODV. The poor performances of DSR are mainly attributed to aggressive use of caching, and lack of any mechanism to expire stale routes or determine the freshness of routes when multiple choices are available. Forceful caching, however, seems to help DSR at low loads and also keeps its routing load down.

\section{FUTURE WORK}

In upcoming years, extensive complex simulations may possibly be carried out by means of the project code, in order to increase a more in-depth performance analysis of the adhoc routing protocols. Further new protocol performance could be studied too.

\section{REFERENCES}

[1] NS-2, The ns Manual (formally known as NS Documentation) available at $h t t p: / / w w w$. isi.edu/nsnam/ns/doc.

[2] Chakeres, Ian \& Belding, Elizabeth. AODV Routing Protocol Implementation Design. Proceedings of the 24th International Conference on Distributed Computing systems Workshops. (2004). 698-703. 10.1109/ICDCSW.2004.1284108.

[3] Perkins, Charles E. and Pravin Bhagwat. "Highly Dynamic Destination-Sequenced Distance-Vector Routing (DSDV) for mobile computers." SIGCOMM (1994).

[4] Johnson, David \& Maltz, David \& Broch, Josh. DSR: The Dynamic Source Routing Protocol for Multi-Hop Wireless Ad Hoc Networks. Ad Hoc Networking. 5 (2002).

[5] Frodigh, Magnus et al. "Wireless ad hoc networking-The art of networking without a network." (2000).

[6] Corson, M.S., \& Park, V.D. Temporally Ordered Routing Algorithm (TORA) version 1: Functional specification" Internet-Draft. (1997).

[7] Fang, Gao \& Yuan, Lu \& Qingshun, Zhang \& Chunli, Li. Simulation and Analysis for the Performance of the Mobile Ad Hoc Network Routing Protocols. (2007). 10.1109/ICEMI.2007.4350745..

[8] Prabhakar Naidu R and Prof. Padmavathamma M, An Architectural Approach to Provide Security to The LL and CL in Smart Phone ADHOC Networks (SPAN). International Journal of Computer Engineering \& Technology, 8(6), 2017, pp. 1-11.

[9] S. Radha Rammohan. Anomaly Detection in Mobile Adhoc Networks (MANET) using C4.5 Clustering Algorithm. International Journal of Information Technology \& Management Information System (IJITMIS), 7(1), 2015, pp. 01-10.

[10] Bhakti Thakre and S. V. Sonekar, Design and Development of an Algorithmic Approach for Selfish and Malicious Node in Cluster Based Adhoc Wireless Networks, International Journal of Computer Engineering and Technology (IJCET), Volume 5, Issue 1, January (2014), pp. 28-37

[11] Mayur N. Bhurewal and Prof. Ms. Sujata G. Tuppad, Efficient Routing and Channel Assignment in Multichannel Mobile Adhoc Networks, International Journal of Computer Engineering \& Technology (IJCET), Volume 6, Issue 6, June (2015), pp. 37-44. 